\title{
The effect of selective serotonin reuptake inhibitor (SSRI) on pain-related behavior in a rat model of neuropathic pain
}

\author{
Hiroharu Saito · Jun Wakai • Miho Sekiguchi • \\ Shinichi Kikuchi $\cdot$ Shinichi Konno
}

Received: 9 April 2013/Revised: 3 May 2014/Accepted: 21 May 2014/Published online: 5 June 2014

(C) The Author(s) 2014. This article is published with open access at Springerlink.com

\begin{abstract}
Introduction Some antidepressants are effective for treating neuropathic pain independent of any effect on depression. Selective serotonin reuptake inhibitors (SSRIs) are one of the potential agents to treat neuropathic pain. The aims of this study were to compare the effects of SSRI and non-steroidal anti-inflammatory drugs (NSAIDs) on pain-related behavior and expression of cytokines in a rat model of neuropathic pain.

Materials and methods Spinal surgery was performed to apply nucleus pulposus (NP) to the dorsal root ganglion (DRG). NP animals were treated with saline $(\mathrm{NP}+\mathrm{S})$, meloxicam $(\mathrm{NP}+\mathrm{M})$, or low-dose or high-dose paroxetine $(\mathrm{NP}+\mathrm{PL}$ and $\mathrm{NP}+\mathrm{PH})$, respectively. Behavioral testing was performed to investigate the mechanical withdrawal thresholds. The numbers of TNF-immunoreactive (IR) neurons in the DRG and of Iba1-IR microglia in the spinal cord (SC) were evaluated using immunohistochemistry. Expression of TNF in the DRG was examined using Western blots.

Results The thresholds on days 14, 21, and 28 were higher in the drug-treated animals than in the $\mathrm{NP}+\mathrm{S}$ group $(p<0.05)$. The number of TNF-IR neurons in DRGs from the NP + M group increased on day 2 and decreased on day 7, and TNF expression in DRGs was significantly higher in the $\mathrm{NP}+\mathrm{S}$ group than in the $\mathrm{NP}+\mathrm{M}$ group on
\end{abstract}

H. Saito · M. Sekiguchi · S. Kikuchi · S. Konno

Department of Orthopaedic Surgery, Fukushima Medical

University School of Medicine, Fukushima, Japan

J. Wakai $\cdot$ M. Sekiguchi $(\bowtie)$

Laboratory Animal Research Center, Fukushima Medical

University School of Medicine, 1-Hikarigaoka, Fukushima,

Fukushima 960-1295, Japan

e-mail: miho-s@fmu.ac.jp days 7,14 . The number of Iba1-IR microglia in the SC was significantly higher in the $\mathrm{NP}+\mathrm{S}$ group than in the $\mathrm{NP}+\mathrm{M}, \mathrm{NP}+\mathrm{PL}$, and $\mathrm{NP}+\mathrm{PH}$ groups on days 7 and 14.

Conclusion An antidepressant might be a potential agent to treat lumbar disc herniation as well as NSAIDs.

Keywords Lumbar disc herniation - Nucleus pulposus . Cox-2 inhibitor · Antidepressant · Tumor necrosis factor-alpha (TNF-alpha) · Microglia

\section{Introduction}

Lumbar disc herniation (LDH) is one of the most common causes of low back pain and sciatica. Herniated discs induce sciatica by both mechanical and chemical factors [1, $13,15,16,29-32,34]$. Proinflammatory cytokines, such as tumor necrosis factor-alpha (TNF- $\alpha)$, Interleukin-1-beta (IL-1 $\beta$ ), Interleukin-6 (IL-6), and nitric oxide, phospholipase A2, and serotonin, are known chemical inducers of sciatica $[1,3,13,14,17,18,22,32]$. Nucleus pulposus contains TNF- $\alpha$, and nucleus pulposus applied to the dorsal root ganglion (DRG) induces pain-related behavior in rats and causes morphological and functional changes in DRG and spinal cord [13, 14, 26, 29, 30, 32, 37, 48].

Neuropathic pain is a sensory disorder caused by the nerve damage. It is characterized by hyperalgesia, spontaneous pain, and allodynia [35]. Various medications are used to treat neuropathic pain. Non-steroidal anti-inflammatory drugs (NSAIDs) are generally used as analgesic drugs, but they are not always effective. Some antidepressants are effective for treating neuropathic pain independent of any effect on depression [36]. Tricyclic antidepressants (TCAs), selective serotonin reuptake 
inhibitors (SSRIs), serotonin-noradrenaline reuptake inhibitors (SNRIs), and other antidepressants differ in their mechanisms of action in the treatment of depression. However, the mechanisms of action of antidepressant drugs in the treatment of neuropathic pain remain uncertain, and the effect of antidepressants compared with those of NSAIDs has not been studied sufficiently.

The purpose of this study was to compare the effects of meloxicam (an NSAID) and paroxetine (SSRI) on painrelated behavior and expression of cytokines in a rat model of neuropathic pain.

\section{Materials and methods}

The animal experiments were carried out under the oversight of the Animal Care and Use Committee in accordance with Guidelines for the Animal Experiments of Fukushima Medical University and the Japanese Government Law Concerning the Protection and Control of Animals.

\section{Animal and anesthesia}

A total of 250 adult female Sprague-Dawley rats (Japan SLC, Shizuoka, Japan) weighing 180-250 g were used. The animals were housed in plastic cages at room temperature in a 12-h light-dark cycle with free access to food and water. Prior to surgery, the animals were anesthetized by intraperitoneal injection of $30 \mathrm{mg} / \mathrm{kg}$ sodium pentobarbital (Nembutal $50 \mathrm{mg} / \mathrm{ml}$; Abbott Laboratories, North Chicago, IL, USA).

\section{Surgical procedure}

Rats were divided into three surgical groups: NP, SF, and sham. Each rat was placed in the prone position, and an incision was made in the middle of the spine at the L4-L6 level. Using a surgical microscope, the fascia was cut, and the multifidus muscles were moved laterally to expose the facet joint between the L5 and 6 lumbar vertebrae. The left L5 nerve root, DRG, and spinal nerve were exposed by L5/ 6 partial laminectomy. For rats in the NP group, nucleus pulposus (NP) harvested from the tail was applied to the DRG ( $n=165)$. For rats in the SF group, the same surgical procedure was performed and subcutaneous fat (SF) tissue, rather than nucleus pulposus, was applied to the DRG $(n=43)$. For rats in the sham group, the same surgical procedure was performed, but no nucleus pulposus or fat tissue was applied to the DRG $(n=55)$. In all groups, the spinal muscles were sutured, and the skin was closed using metal clips.
Drug treatments in the NP groups

Animals from the NP group were divided into five treatment groups: $\mathrm{NP}+\mathrm{S}$ (saline) group, $\mathrm{NP}+\mathrm{M}(0.2 \mathrm{mg} / \mathrm{kg}$ meloxicam) group, $\mathrm{NP}+\mathrm{PL}$ (low-dose paroxetine; $0.2 \mathrm{mg} / \mathrm{kg}$ ) group, $\mathrm{NP}+\mathrm{PH}$ (high-dose paroxetine; $0.8 \mathrm{mg} / \mathrm{kg}$ ) group, and $\mathrm{NP}+\mathrm{M}+\mathrm{PH}$ (combination $0.2 \mathrm{mg} / \mathrm{kg}$ meloxicam $+0.8 \mathrm{mg} / \mathrm{kg}$ paroxetine) group. Meloxicam (NSAIDs) is a cyclooxygenase-2 (COX-2) inhibitor. Paroxetine is a selective serotonin reuptake inhibitor (SSRI) used as an antidepressant. $0.9 \%$ saline was used for administration and to dissolve the drugs. The concentrations of each drug were based on those used in the clinical setting. Animals received $0.3 \mathrm{ml}$ of the designated treatment solution orally once daily for 10 days beginning 1 day after surgery.

\section{Behavioral testing}

Behavioral tests were performed by a physician who was blinded to the experimental groups ( $n=12$ each group) before and after surgery that we showed following two experiment. Sensitivity to non-noxious mechanical stimuli was tested using the von Frey test. Baseline testing was performed before the start of the experiment to accommodate animals with normal responses. Hind paw withdrawal response to von Frey hair (North Coast Medical, Morgan Hill, CA, USA) stimulation of the plantar surface of the footpads was determined at 2, 7, 14, 21, 28, and 35 days after surgery. Individual rats were placed in an acrylic cage with a mesh floor and allowed to acclimate for $15 \mathrm{~min}$ or until cage exploration and major grooming activities ceased. The lateral plantar surface of the operated hind paw, innervated by the L5 nerve, was stimulated with 9 von Frey filaments $(1.02,1.4,2.0$, 4.1, 6.1, 8.0, 10.6, 15.4, and $26.0 \mathrm{~g}$ ) threaded under the mesh floor. The gram ratings for von Frey hairs were based on the ratings supplied by the manufacturer. Stimulation was initiated with the 1.02-g filament. The filament was sequentially applied to the paw surface just until the filament bent and was held for approximately $3 \mathrm{~s}$. The response was considered positive if the rat lifted the foot in combination with either licking or shaking of the foot as an escape response.

Experiment 1 The von Frey test was performed to investigate the effect of weight of harvested fat and NP on nerve in the NP, SF, and sham groups.

Experiment 2 The von Frey test was performed on rats in the $\mathrm{NP}+\mathrm{S}, \quad \mathrm{NP}+\mathrm{M}, \quad \mathrm{NP}+\mathrm{PL}, \quad \mathrm{NP}+\mathrm{PH}, \quad$ and $\mathrm{NP}+\mathrm{M}+\mathrm{PH}$ groups to assess the effects of meloxicam, paroxetine, and the meloxicam-paroxetine cocktail. 
Immunohistochemical study

Immunohistochemical examinations were performed 2, 7, and 14 days after surgery ( $n=5$ rats for each group at each time point). The rats were anesthetized using $99 \%$ diethyl ether (Wako Pure Chemical Industries, Osaka, Japan), perfused with fresh $4 \%$ paraformaldehyde in $0.1 \mathrm{M}$ phosphate-buffered saline (PBS), and the left L5 DRG and the L5 segment of the spinal cord (SC) were removed. The DRG and SC were postfixed in $4 \%$ paraformaldehyde and subsequently embedded in paraffin. Two sections ( $6 \mu \mathrm{m}$ thick) were cut from each DRG and each SC and placed individually on separate slides. The following antibodies were used. To recognize satellite glial cells, glial fibrillary acidic protein (GFAP) was used. To recognize endoneurial macrophages, OX-6 was used. To recognize nuclei in neurons, NeuN was used. To recognize microglia, ionized calcium-binding adapter molecule 1 (Iba1) was used. Each sample was also double labeled using anti-TNF $\alpha$ antibodies. GFAP, OX-6, and NeuN antibody were used on DRG samples, and Iba-1 antibody was used on SC samples. Sections were deparaffinized with xylene and rehydrated with $100 \%$ ethanol followed by PBS, and pretreated with Dako Target Retrieval Solution (Dako North America, Carpinteria, CA, USA) at $97{ }^{\circ} \mathrm{C}$ for 20 min to enhance immunoreactivity. Samples were incubated in $2 \%$ normal donkey serum in PBS/ $0.3 \%$ Triton $\mathrm{X}-100$ applied for $1 \mathrm{~h}$ at room temperature; goat anti-rat TNF $\alpha$ antibody (1:100; R\&D Systems, Minneapolis, MN, USA) was applied, and samples were incubated overnight at $4{ }^{\circ} \mathrm{C}$, after washing step by PBS, followed by incubation for $1 \mathrm{~h}$ at room temperature with donkey anti-goat Alexa Fluor 555 (red) fluorescent antibody (1:200; Molecular Probes, Eugene, OR, USA). Next, samples were incubated in $2 \%$ normal goat serum in PBS/0.3 \% triton X-100 for $1 \mathrm{~h}$. Samples then were incubated with mouse anti-GFAP (1:200; Millipore, Billerica, MA, USA), rabbit anti-Iba1 (1:200; Wako, Osaka, Japan), mouse anti-OX-6 (1:100, Serotec, Kidlington, UK), or mouse anti-NeuN (1:100, Millipore) antibody overnight at $4{ }^{\circ} \mathrm{C}$. The sections were rinsed in PBS and incubated for $1 \mathrm{~h}$ at room temperature with goat antimouse or goat anti-rabbit Alexa Fluor 488 (green) (1:200; Molecular Probes). After rinsing, the sections were mounted on microscope slides with VECTASHIELD mounting medium containing DAPI (H-1200, Vector, Burlingame, CA, USA). DAPI (4', 6-diamidino-2-phenylindole) specifically stains nuclei, with little or no cytoplasmic labeling. The blue fluorescence of 4', 6-diamidino-2-phenylindole stands in vivid contrast to green fluorescent probes was used to label other structures. Fluorescent staining was analyzed using a BX50 fluorescent microscope (Olympus, Tokyo, Japan) equipped with Axio Vision imaging software (Carl Zeiss, Gottingen, Germany). Five slides of each DRG and SC were used to determine the numbers of TNF $\alpha$-immunoreactive (IR) neurons and Iba-1-IR microglia. The mean number of TNF $\alpha$ IR neurons in an area of 100 DRG neurons, and the mean number of Iba-1-IR microglia in a $1 \times 10^{5} \mu \mathrm{m}^{2}$ area in the left dorsal horn were determined.

Immunoblot analyses

Immunoblot analyses assessing protein expression were performed 2, 7, and 14 days after surgery in the NP $+\mathrm{S}$, $\mathrm{NP}+\mathrm{M}, \mathrm{NP}+\mathrm{PL}, \mathrm{NP}+\mathrm{PH}, \mathrm{SF}$, and sham groups $(n=4$, for each time point). Naive rats that had not undergone surgery were used as controls ( $n=4$, from each group, for each time point). The rats were rapidly decapitated under anesthesia, and the left L5 DRGs were removed and frozen in liquid nitrogen. Samples were homogenized in lysis buffer (\#9803; Cell Signaling, Danvers, MA, USA). Protein concentration of each sample was measured using a bicinchoninic acid protein assay kit (Pierce, Rockford, IL, USA). Total protein ( $20 \mathrm{mg} / \mathrm{lane}$ ) was run on $10 \%$ tris-glycine SDS-polyacrylamide gel electrophoresis (Bio-Rad, Hercules, CA, USA) for $1.5 \mathrm{~h}$ at 10-40 mA and then transferred to polyvinylidene difluoride filter membranes (Millipore) for $1 \mathrm{~h}$ at $15 \mathrm{~V}$. The membranes were incubated with primary antibody diluted in $5 \%$ nonfat dry milk, $1 \times$ tris-buffered saline, and $0.1 \%$ tween- 20 at $4{ }^{\circ} \mathrm{C}$ with gentle shaking overnight. Primary antibodies used for incubation were mouse anti-TNF- $\alpha$ (1:100; R\&D Systems) and mouse anti- $\beta$ actin (1:5000; Sigma, St. Louis, MO, USA). The membranes were incubated for $1 \mathrm{~h}$ at room temperature with horseradish peroxidase-conjugated secondary antibody (1:5000; Bio-Rad). Positive bands were visualized using an enhanced chemiluminescence system (Amersham Bioscience, Piscataway, NJ, USA). Signal from TNF- $\alpha$-positive bands were measured relative to signal from the internal controls, $\beta$-actin-positive bands, using an imaging analysis system (Image J, version 1.38u; National Institute of Mental Health, Bethesda, MD, USA). In addition, each band was calculated as the ratio to the control group.

Statistical analysis

All data are reported as mean \pm standard deviation (SD). Statistical differences among multiple groups were assessed using the Bonferroni test. $p$ values less than 0.05 were considered significant.

\section{Results}

Behavioral testing

\section{Experiment 1}

In the NP group, the mechanical withdrawal threshold decreased 2 days after surgery. In the SF and sham 
Fig. 1 Changes in mechanical withdrawal threshold of the foot pad in rats. From days 2 to 28, the mechanical withdrawal threshold was significantly lower in the NP group than in the $\mathrm{SF}$ and sham groups $(p<0.05)$. There were no significant differences between the SF and sham groups

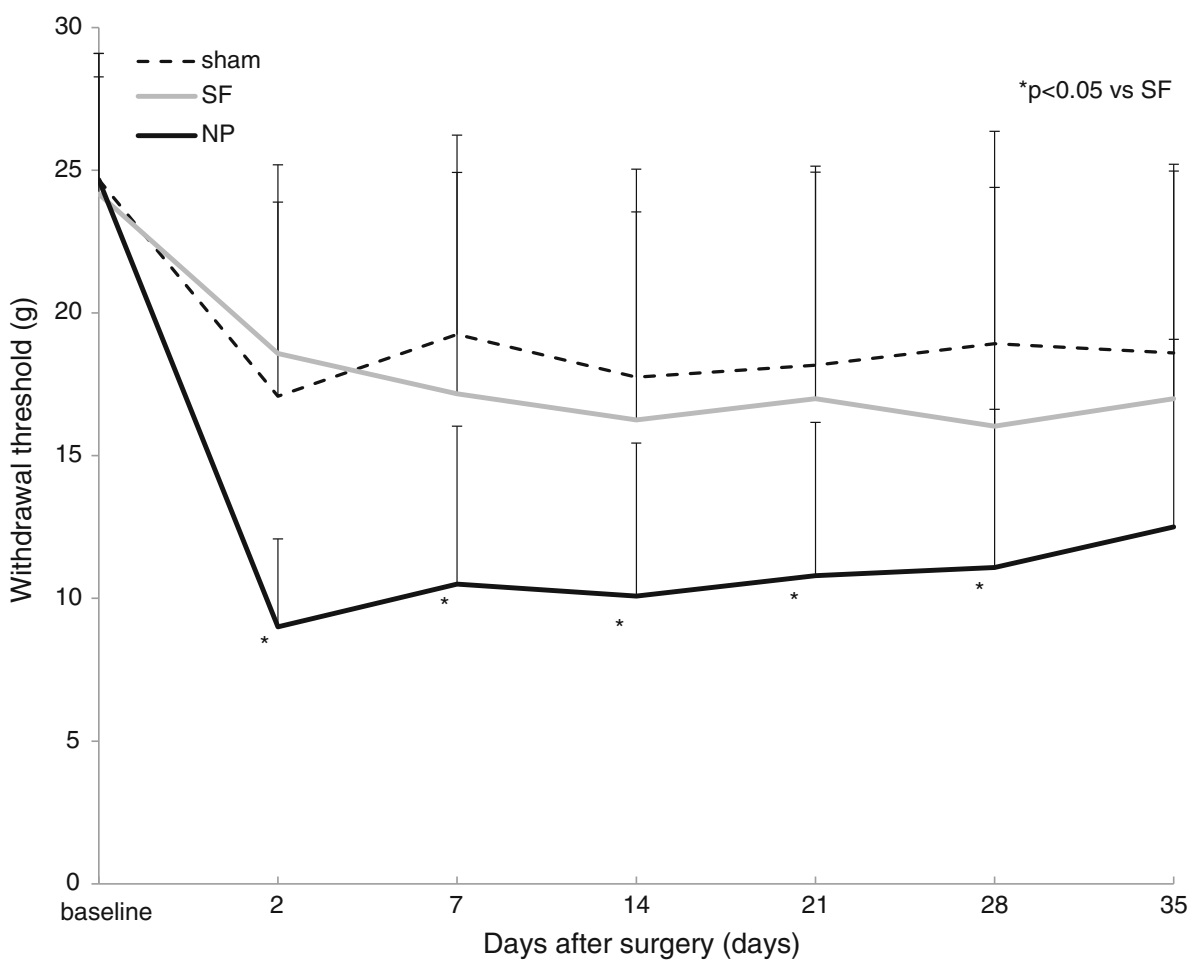

Fig. 2 Changes in mechanical withdrawal threshold after drug or saline treatments. From days 2 to 28 , the mechanical withdrawal threshold was significantly lower in the $\mathrm{NP}+\mathrm{S}$ group than in the sham group $(p<0.01)$. In the $\mathrm{NP}+\mathrm{M}, \mathrm{NP}+\mathrm{PL}, \mathrm{NP}+\mathrm{PH}$, and $\mathrm{NP}+\mathrm{M}+\mathrm{PH}$ groups, the thresholds were low until day 7 ; but from days 14 to 28 the thresholds in the drug-treatment groups were higher than that in the NP $+\mathrm{S}$ group $(p<0.05)$.

There was significant difference between the NP + PL and $\mathrm{NP}+\mathrm{PH}$ groups

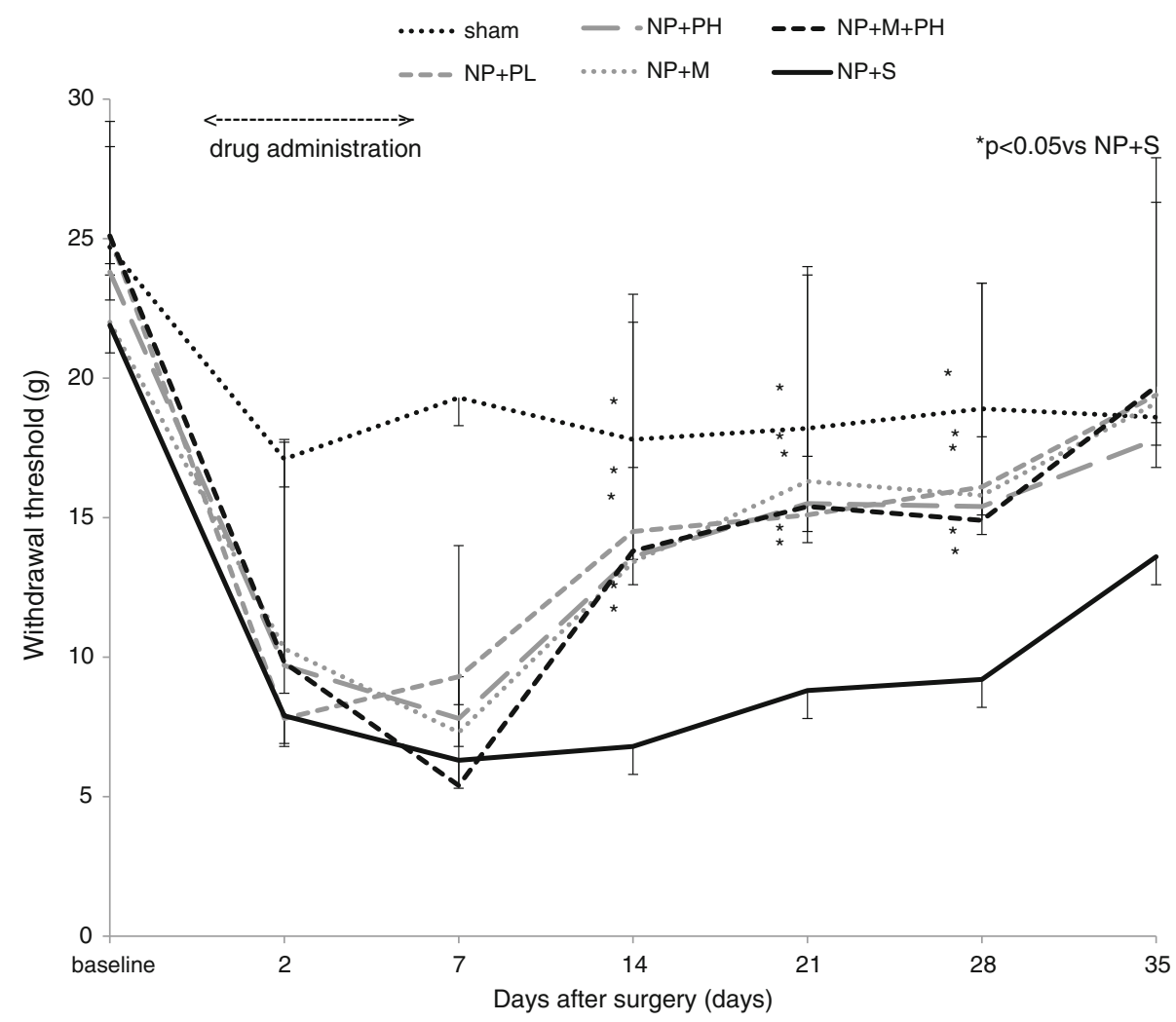

groups, the threshold did not decrease. From day 2 to day 28, the threshold was significantly lower in the NP group than in the SF or sham group $(p<0.05)$ (Fig. 1). The thresholds in the SF and sham groups were not significantly different at any time point. These findings indicated that the decrease of threshold in the NP group did not result from compression of fat as a mechanical factor. 


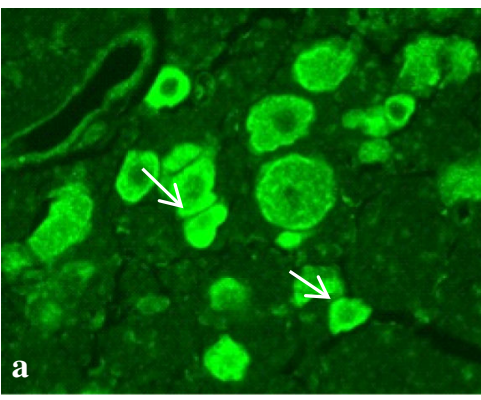

TNF $\alpha$

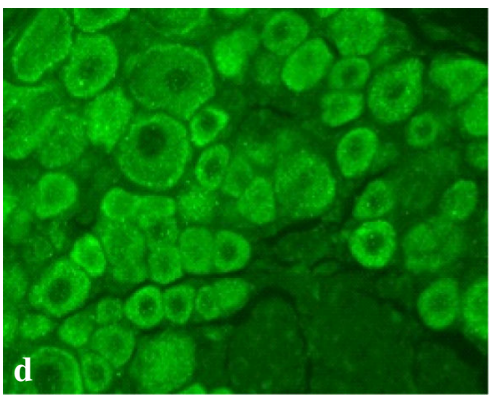

TNF $\alpha$

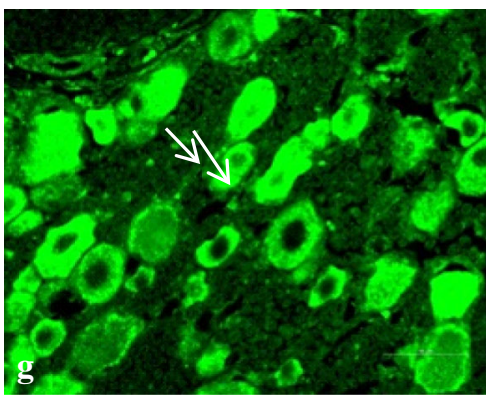

TNF $\alpha$

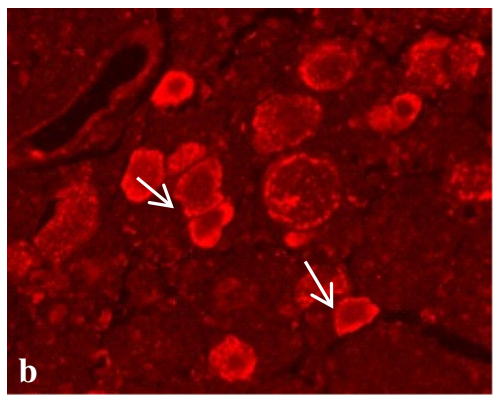

GFAP

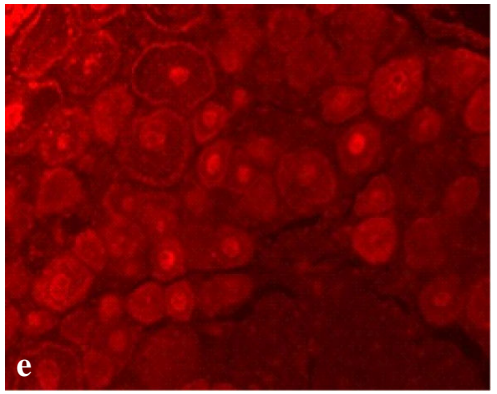

NeuN

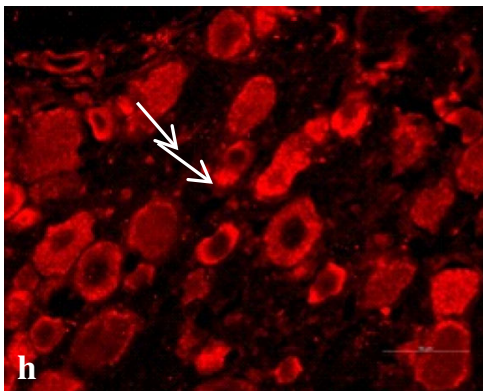

OX-6

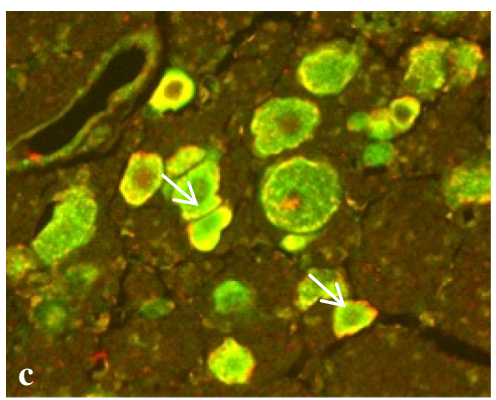

merged

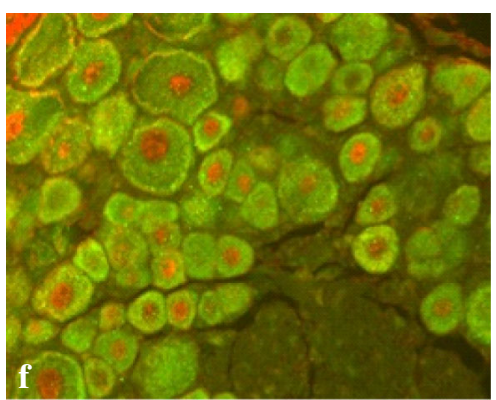

not merged

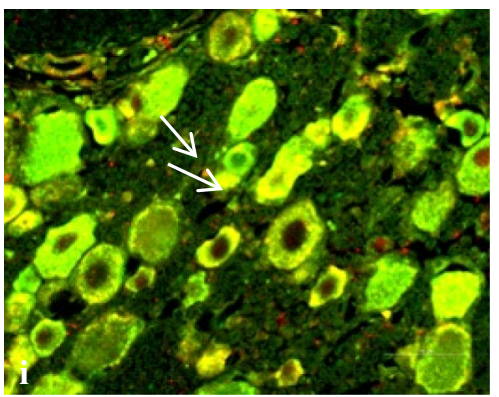

merged
Fig. 3 TNF $\alpha$-IR neurons in DRG in the NP $+S$ group (day 2). TNF was expressed in the soma of neurons. TNF $\alpha$ signal (green) (a, d, g) co-localized with GFAP-positive satellite glial cells (b) and OX-6-

\section{Experiment 2}

In the NP $+\mathrm{S}$ group, the mechanical withdrawal thresholds decreased by day 2 and remained low until day 28. In the $\mathrm{NP}+\mathrm{M}, \mathrm{NP}+\mathrm{PL}, \mathrm{NP}+\mathrm{PH}$, and $\mathrm{NP}+\mathrm{M}+\mathrm{PH}$ groups, the threshold decreased by day 2 and remained low until day 7 , but from 14 to 28 days after surgery, the threshold was higher in each of the drug-treatment groups compared to that in the NP $+\mathrm{S}$ group $(p<0.05)$ (Fig. 2).

Immunohistochemical analyses

Images of immunostained DRG sections from the NP $+\mathrm{S}$ group at day 2 are shown in Fig. 3. TNF (green) was expressed in the soma of DRG neurons. TNF (green) colocalized with GFAP-positive satellite glial cells (red), and positive macrophages (h), but did not co-localized with NeuNpositive neurons (e) in the NP $+\mathrm{S}$ group at day 2. Scale bar $50 \mu \mathrm{m}$
OX-6 (red) positive macrophages, but it did not co-localized with NeuN (red) positive cells. Images of immunostained SC sections from the NP $+\mathrm{S}$ group at day 7 are shown in Fig. 4. TNFa (red) co-localized with Iba-1 positive microglia (green). The number of TNF $\alpha$-IR neurons was higher in the NP $+\mathrm{S}$ (no drug) group than in the sham group $(p<0.01)$. The numbers of TNF $\alpha$-IR neurons in the SF and sham groups were not significantly different (data was not shown). In the NP $+\mathrm{M}$ group, the number of TNF $\alpha$-IR neurons of DRGs decreased after days 7 and 14 significantly compared with the $\mathrm{NP}+\mathrm{S}$ group, respectively $(p<0.05)$. There was no significant difference among the $\mathrm{NP}+\mathrm{PL}, \mathrm{NP}+\mathrm{PH}$ and $\mathrm{NP}+\mathrm{S}$ groups in the number of TNFa-IR neurons (Fig. 5). The number of Iba1IR microglia in the dorsal horn increased, and there was no significant difference among the three treatment groups on 


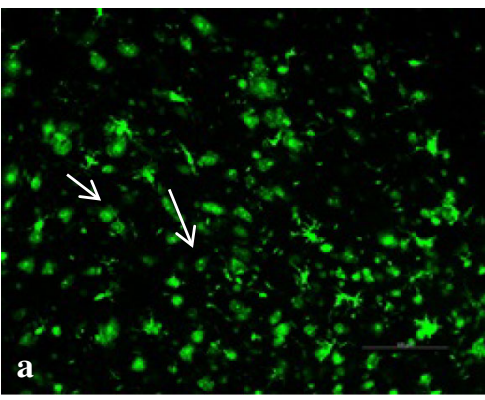

Iba-1

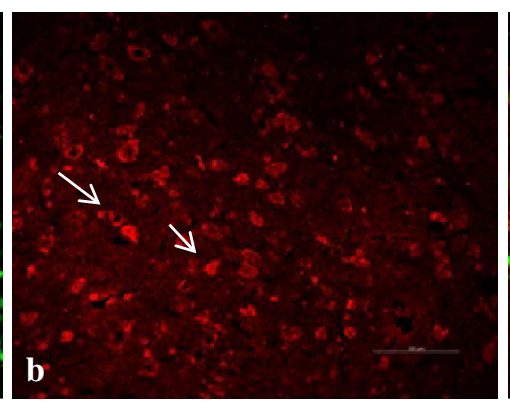

TNF $\alpha$

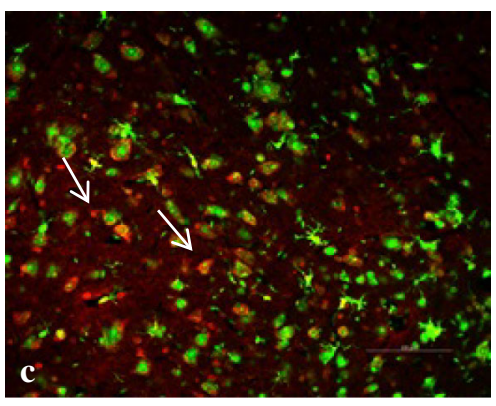

merged

Fig. 4 Iba1-IR migroglia in SC in the NP $+\mathrm{S}$ group (day 7). Iba-IR migroglia (green) (a) co-localized with TNF- $\alpha$ (red) (b). Scale bar $100 \mu \mathrm{m}$

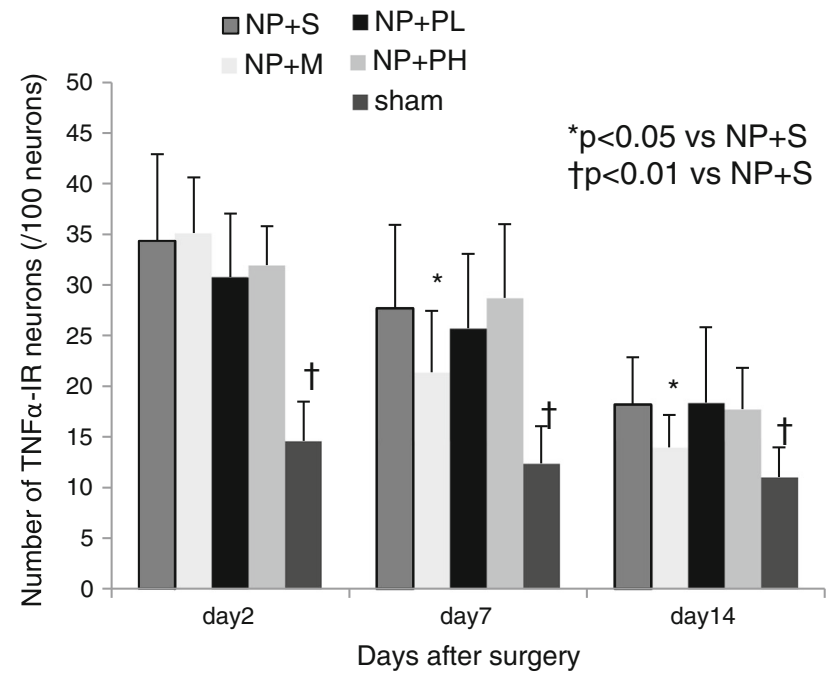

Fig. 5 The number of TNF $\alpha$-IR neurons in DRG. On days 7 and 14, the number of TNF $\alpha$-IR neurons was significantly lower in the $\mathrm{NP}+\mathrm{M}$ group than the $\mathrm{NP}+\mathrm{S}$ group $(p<0.05)$. There was no significant difference in TNF $\alpha-I R$ among the NP $+\mathrm{PL}, \mathrm{NP}+\mathrm{PH}$, and $\mathrm{NP}+\mathrm{S}$ groups

day 2 (Fig. 6). In the NP $+\mathrm{M}, \mathrm{NP}+\mathrm{PL}$, and $\mathrm{NP}+\mathrm{PH}$ groups, the numbers of Iba1-IR microglia on day 7 and day 14 were significantly lower than in the NP $+\mathrm{S}$ group $(p<0.05)$.

\section{Immunoblotting for TNF}

According to the behavioral testing and immunohistochemical analysis, there was no concentration dependence between the NP + PL and NP + PH groups. Therefore, $0.2 \mathrm{mg} / \mathrm{kg}$ (PL), rather than $0.8 \mathrm{mg} / \mathrm{kg}(\mathrm{PH})$, paroxetine was used in the immunoblotting analysis.

TNF $\alpha$-positive bands in the DRG samples were detected at $26 \mathrm{kDa}$. There was no significant difference in the expression level of $\mathrm{TNF} \alpha$ among the $\mathrm{NP}+\mathrm{S}, \mathrm{NP}+\mathrm{M}$, and NP + PL groups at day 2 (Fig. 7). TNF $\alpha$ expression was significantly higher in the $\mathrm{NP}+\mathrm{S}$ group than in the

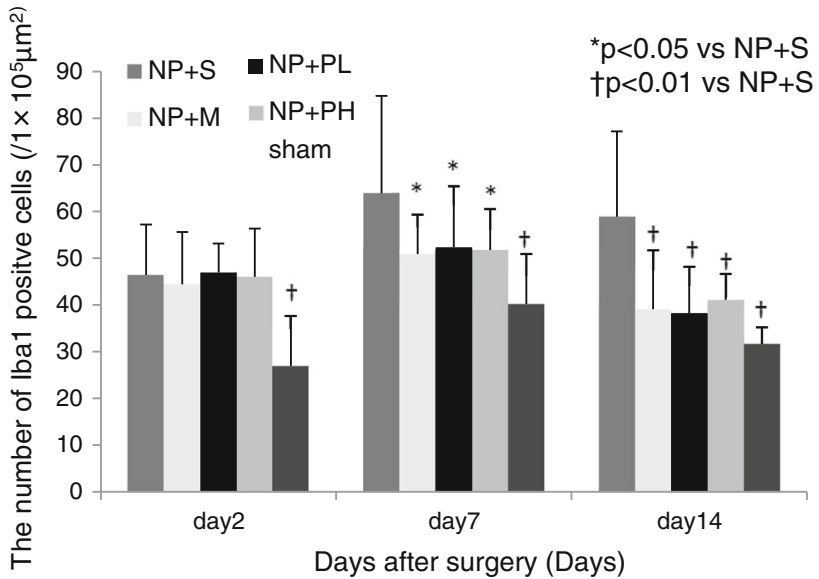

Fig. 6 The number of Iba1-IR microglia in SC. On days 7 and 14, the number of Iba1-IR microglia was significantly higher in the NP $+\mathrm{S}$ group than the NP $+\mathrm{M}, \mathrm{NP}+\mathrm{PL}$, and NP $+\mathrm{PH}$ groups $(p<0.05)$. There was no significant difference among groups at day 2

sham group 14 days after surgery. TNF $\alpha$ expression was significantly lower in the $\mathrm{NP}+\mathrm{M}$ group than in the $\mathrm{NP}+\mathrm{S}$ group 7 and 14 days after surgery $(p<0.01)$.

\section{Discussion}

Here, we first investigated whether the NP-applied rat model used shows a predominantly mechanical or chemical effect. Olmarker et al. reported that epidural application of autologous NP to the sacrococcygeal cauda equina induced nerve fiber degeneration and marked reduction in nerve conduction velocity in spinal nerve roots, but application of retroperitoneal fat did not [4]. To evaluate the influence of mechanical effects harvested NP in this study, an equal amount of fat was harvested and applied in the SF animal group. Although subcutaneous fat, rather than retroperitoneal fat was used, there was no significant difference in results of the behavioral tests or expression of TNF $\alpha$ in DRG or SC between the SF and sham groups. These 


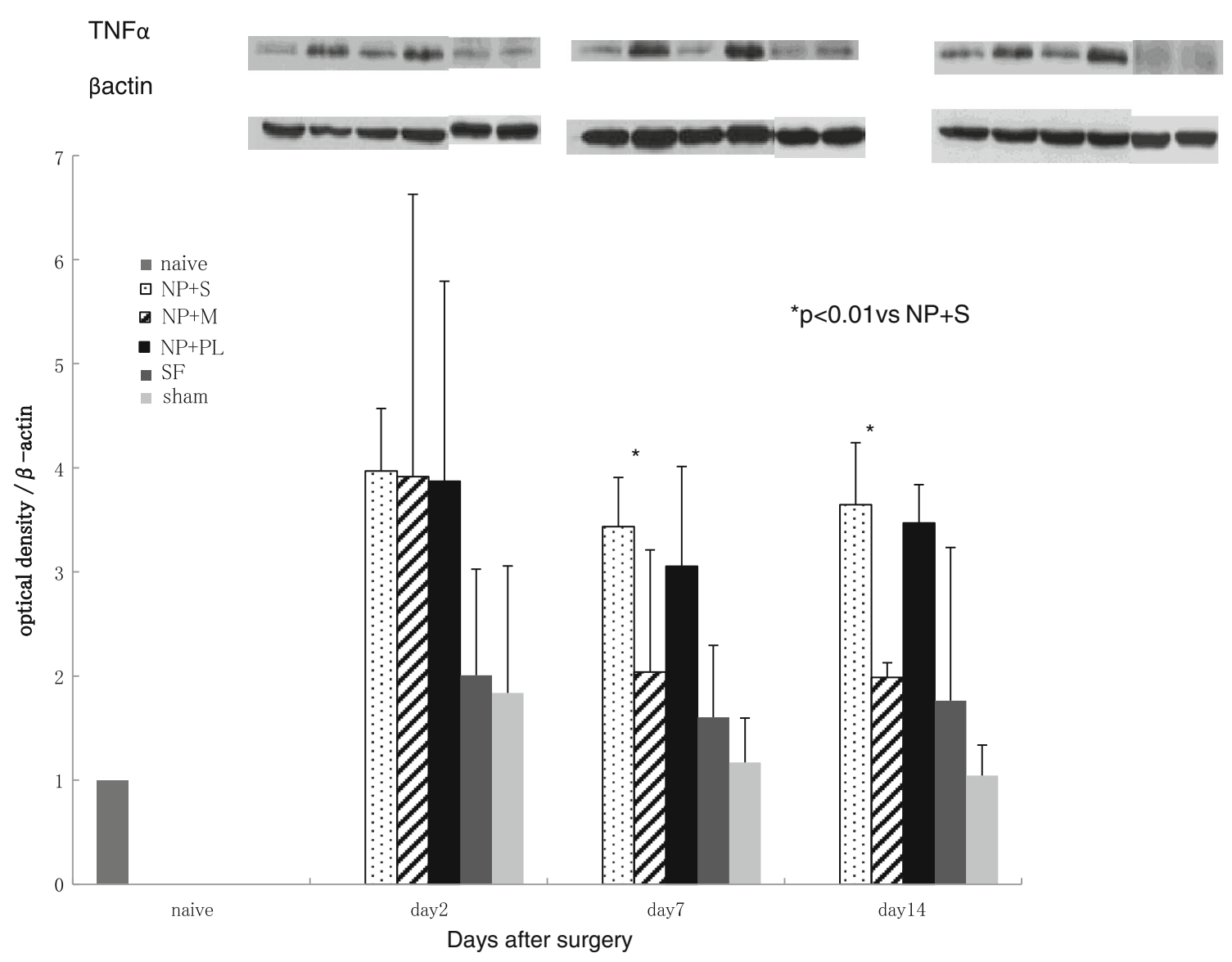

Fig. 7 Expression of TNF $\alpha$ protein in the left L5 DRG. TNF $\alpha$-positive bands derived from DRG sample were detected at $26 \mathrm{kDa}$. On days 7 and 14 , expression of TNF was significantly lower in the NP $+\mathrm{M}$ group than the NP $+\mathrm{S}$ group $(p<0.01)$

findings indicate that the amount of NP in the NP group does not have a mechanical impact on the nerve.

Next, we compared the effects of an SSRI and an NSAID on pain-related behavior and cytokine expression. In the behavioral test, the mechanical withdraw thresholds of all drug-treatment groups improved from days 14 to 28 relative to those of the saline-treatment group. On days 7 and 14, the number of TNF $\alpha$-IR cells and the level of $\mathrm{TNF} \alpha$ expression in DRGs were lower in NSAID-treated animals (NP $+\mathrm{M}$ group) than in saline-treated animals $(\mathrm{NP}+\mathrm{S}$ group). While there were no changes in the number of TNF $\alpha$-IR cells and levels of TNF $\alpha$ expression in the NP + PL group after SSRI administration.

Recently, it was reported that the $\mathrm{P} 2 \mathrm{X} 4$ receptor expressed in activated microglia is related with neuropathic pain $[19,45]$. The P2X4 receptor is a ligand-gated ionchannel receptor that is activated by ATP $[5,20,21]$. The cells expressing $\mathrm{P} 2 \mathrm{X} 4$ receptors are microglia, rather than neurons or astrocytes. Upon peripheral nerve injury, ATP released from primary afferent central terminals in the dorsal horn of the spinal cord modulates neurotransmission in the glutamatergic and GABAergic systems in dorsal horn neurons $[12,24,28]$. Thus, activation of the P2X4 receptor in the spinal cord causes pain behavior $[6,44]$. Recent studies indicate that paroxetine inhibits P2X4 receptors expressed in microglia $[27,45]$.

Here, from days 7 to 14 after surgery, the number of IbaIR cells (i.e., microglia) in the SC was lower in paroxetinetreated animals than in saline-treated animals. Powerful inhibition of $\mathrm{P} 2 \mathrm{X} 4$ receptors may be responsible for the analgesic effect of paroxetine, and it is possible that some antidepressants used to treat patients with neuropathic pain produce antiallodynic effects, at least in part, via inhibitory effects on P2X4 receptors [41]. Inhibitory effects on P2X4 receptors indicate that paroxetine might affect the SC and improve pain-related behavior.

In contrast, the number of Iba1-IR cells decreased after administration of an NSAID in the present study. In peripheral nerve injury, such as LDH, TNF $\alpha$ expression is upregulated in endoneurial macrophages and Schwann cells, resulting in pain $[46,47] \mathrm{TNF} \alpha$ produced at nerve injury sites is axonally transported to DRG neurons and 
the spinal cord dorsal horn. TNF $\alpha$ signaling may activate central cytokines in the pathogenesis of painful neuropathy $[38,39]$. Once activated by peripheral and central nerve injury, microglia change in form, number, function, and gene expression [7, 23, 42, 43]. Microglia usually act to eliminate microorganisms and deleterious debris and may be involved in neuroprotection by producing neurotrophic factors [9, 41]. Microglia also have a cytotoxic function because they release reactive oxygen species, nitric oxide, or inflammatory cytokines; these released factors produce immunological actions and act on neurons to alter their function $[2,4,8,10,11,23,40,43]$. NSAIDs might affect the SC indirectly through signals from the DRG or direct suppression of cytokines expression in spinal microglia. A pain-related behavior maintains after decrease of TNF expression. This result suggested that other factors influence a lasting longer. In this study, activated microglia, which are related with neuropathic pain, were focused on. Therefore, we did not investigate the expression of TNF in the SC after drug treatment was not investigated in this study. However, one of the limitations of this study was effect of NSAIDs and SSRI on TNE expression in SC.

Results from this study demonstrated that SSRI, paroxetine, improved pain-related behavior in the NP-applied rat model by suppressing microglia. Based on our previous study using a NP rat model, P2X4 receptors are expressed in endoneurial macrophages in the DRG [33]. An effect of paroxetine on the DRG was not observed in the present study. Additionally, a quantitative analysis of microglia in the SC was not conducted following application of NP and subsequent drug or saline treatments.

Another limitation of the present study was that astrocytes were not evaluated. Activation of microglia precedes activation of astrocyte [25], and astrocytes may be associated with the maintenance of allodynia. Further more studies are needed to investigate tendency and interaction with microglia in astrocytes.

In conclusion, paroxetine, SSRI, had an effect on a painrelated behavior that resulted from the application of NP to the DRG. In the clinical situation, early recovering from neuropathic pain due to lumbar disc herniation might be expected using SSRI.

Acknowledgments The authors thank Akira Sato. Rie Shibuya and Yumi Takakura for their technical assistance.

\section{Conflict of interest None.}

Open Access This article is distributed under the terms of the Creative Commons Attribution License which permits any use, distribution, and reproduction in any medium, provided the original author(s) and the source are credited.

\section{References}

1. Aoki Y, Rydevik B, Kikuchi S et al (2002) Local applications of disc-related cytokines on spinal nerve roots. Spine 27:1614-1617

2. Boje KM, Arora PK (1992) Microglial-produced nitric oxide and reactive nitrogen oxides mediate neuronal cell death. Brain Res 587:250-256

3. Brisby H, Byröd G, Olmarker K et al (2000) Nitric oxide as a mediator of nucleus pulposus-induced effects on spinal nerve roots. J Orthop Res 18:815-820

4. Calingasan NY, Park LC, Calo LL et al (1998) Induction of nitric oxide synthase and microglial responses precede selective cell death induced by chronic impairment of oxidative metabolism. Am J Pathol 153:599-610

5. Chizh BA, Illes P (2001) P2X receptors and nociception. Pharmacol Rev 53:553-568

6. Driessen B, Reimann W, Selve N et al (1994) Antinociceptive effect of intrathecally administered P2-purinoceptor antagonists in rats. Brain Res 666:182-188

7. Georg WK (1996) Microglia: a sensor for pathological events in the CNS. Trends Neurosci 19:312-318

8. Giulian D, Baker TJ, Shih LC et al (1986) Interleukin 1 of the central nervous system is produced by ameboid microglia. J Exp Med 164:594-604

9. Hamanoue M, Takemoto N, Matsumoto K et al (1996) Neurotrophic effect of hepatocyte growth factor on central nervous system neurons in vitro. J Neurosci Res 43:554-564

10. Hanisch UK (2002) Micoglia as a source and target of cytokines. Glia 40:140-155

11. Olmarker K, Larsson K (1998) Tumor necrosis factor alpha and nucleus pulposus induced nerve root injury. Spine 23:2538-2544

12. Hugel S, Schlichter R (2000) Presynaptic P2X receptors facilitate inhibitory GABAergic transmission between cultured rat spinal cord dorsal horn neurons. J Neurosci 20:2121-2130

13. Igarashi T, Kikuchi S, Shuvayev V et al (2000) Exogenous tumor necrosis factor-alpha mimics nucleus pulposus-induced neuropathology: molecular, histologic, and behavioral comparisons in rat. Spine 25:2975-2980

14. Kato K, Kikuchi S, Konno S et al (2008) Participation of 5-hydroxytryptamine in pain-related behavior induced by nucleus pulposus applied on the nerve root in rats. Spine 33(12): $1330-1336$

15. Kayama S, Konno S, Olmarker K et al (1996) Incision of the annulus fibrosus induces nerve root morphologic, vascular, and functional changes: an experimental study. Spine 21:2539-2543

16. Kawakami M, Tamaki T, Weinstein JN et al (1996) Pathomechanism of pain-related behavior produced by allografts of intervertebral disc in the rat. Spine 21:2101-2107

17. Kawakami M, Tamaki T, Hashizume H et al (1997) The role of phospholipase A2 and nitric oxide in pain-related behavior produced by an allograft of intervertebral disc material to the sciatic nerve of the rat. Spine 22:1074-1079

18. Kawakami M, Tamaki T, Hashizume H et al (1997) The role of phospholipase A2 and nitric oxide in pain-related behavior produced by an allograft of intervertebral disc material to the sciatic nerve of the rat. Spine 22:1074-1079

19. Keller AF, Beggs S, Salter MW et al (2007) Transformation of the output of spinal lamina I neurons after nerve injury and microglia stimulation underlying neuropathic pain. Mol Pain 3:27

20. Kennedy C, Assis TS, Currie AJ et al (2003) Crossing the pain barrier: P2 receptors as targets for novel analgesics. J Physiol 553:683-694

21. Khakh BS, Burnstick G, Kennedy C et al (2001) International union of pharmacology. Current status of the nomenclature and 
properties of P2X receptors and their subunits. Pharmacol Rev 53:107-118

22. Kobayashi H, Kikuchi S, Konno S et al (2011) Interaction of 5-hydroxytryptamine and tumor necrosis factor-alpha to painrelated behavior by nucleus pulposus applied on the nerve root in rats. Spine 36(3):210-218

23. Kreutzberg GW (1996) Microglia: a sensor for pathological events in the CNS. Trends Neurosci 19:312-318

24. Li P, Calejesan AA, Zhuo M (1998) ATP P2X receptors and sensory synaptic transmission between primary afferent fibers and spinal dorsal horn neurons in rats. J Neurophysiol 80:3356-3360

25. Merrill JE (1991) Effects of interleukin-1 and tumor necrosis factor-alpha on astrocytes, microglia, oligodendrocytes, and glial precursors in vitro. Dev Neurosci 13:130-137

26. Miyoshi S, Sekiguchi M, Konno S et al (2011) Increased expression of vascular endothelial growth factor protein in dorsal root ganglion exposed to nucleus pulposus on the nerve root in rats. Spine 36(1):E1-E6

27. Nagata K, Imai $T$, Yamashita $T$ et al (2009) Antidepressants inhibit P2X4 receptor function: a possible involvement in neuropathic pain relief. Mol Pain 5:20

28. Nakatsuka T, Tsuzuki K, Ling JX et al (2003) Distinct roles of $\mathrm{P} 2 \mathrm{X}$ receptors in modulating glutamate release at different primary sensory synapses in rat spinal cord. J Neurophysiol 89:3243-3252

29. Olmarker K, Rydevik B, Nordborg C (1993) Autologous nucleus pulposus induces neurophysiologic and histologic changes in porcine cauda equine nerve roots. Spine 18:1425-1432

30. Olmarker K, Brisby H, Yabuki S et al (1997) The effects of normal, frozen, and hyaluronidase-digested nucleus pulposus on nerve root structure and function. Spine 22:471-476

31. Olmarker K, Myers RR (1998) Pathogenesis of sciatic pain: role of herniated nucleus pulposus and deformation of spinal nerve root and dorsal root ganglion. Pain 78:99-105

32. Olmarker K, Larsson K (1998) Tumor necrosis factor alpha and nucleus pulposus induced nerve root injury. Spine 23:2538-2544

33. Otoshi K, Kikuchi S, Konno S et al (2010) The reactions of glial cells and endoneurial macrophages in the dorsal root ganglion and their contribution to pain-related behavior after application of nucleus pulposus onto the nerve root in rats. Spine 35(3):264-271
34. Peng B, Wu W, Li Z et al (2007) Chemical radiculitis. Pain 127:11-16

35. Ji R-R, Suter MR (2007) P38 MAPK, microglial signaling, and neuropathic pain. Mol Pain 3:33

36. Saarto T, Wiffen P (2010) Antidepressants for neuropathic pain: a chochrane review. J Neurol Neurosurg Phychiatry 81:1372-1373

37. Sekiguchi M, Otoshi K, Kikuchi S et al (2011) Analgesic effects of prostaglandin E2 receptor subtype EP1 receptor antagonist: experimental study of application of nucleus pulposus. Spine 36(22):1829-1834

38. Shubayev VI, Myers RR (2001) Axonal transport of TNF-alpha in painful neuropathy: distribution of ligand tracer and TNF receptors. J Neuroimmunol 114:48-56

39. Shubayev VI, Myers RR (2002) Anterograde TNF alpha transport from rat dorsal root ganglion to spinal cord and injured sciatic nerve. Neurosci Lett 320:99-101

40. Smith ME, van der Maesen K, Somera FP (1998) Macrophage and microglial responses to cytokines in vitro: phagocytic activity, proteolytic enzyme release, and free radical production. J Neurosci Res 54:68-78

41. Streit WJ, Graeber MB, Kreutzberg GW (1998) Functional plasticity of microglia: a review. Glia 1:301-307

42. Streit WJ, Walter SA, Pennell NA (1999) Reactive microgliosis. Prog Neurobiol 57:563-581

43. Stoll G, Jander S (1999) The role of microglia and macrophages in the pathophysiology of the CNS. Prog Neurobiol 58:233-247

44. Tsuda M, Ueno S, Inoue K (1999) In vivo pathway of thermal hyperalgesia by intrathecal administration of $\alpha, \beta$-methylene ATP in mouse spinal cord: involvement of the glutamate-NMDA receptor system. Br J Pharmacol 127:449-456

45. Tsuda M, Shigemoto-Mogami Y, Koizumi S et al (2003) P2X4 receptors induced in spinal microglia gate tactile allodynia after nerve injury. Nature 424:778-783

46. Wagner R, Myers RR (1996) Schwann cells produce TNF-alpha: expression in injured and non-injured nerves. Neuroscience 73:625-629

47. Wagner R, Myers RR (1996) Endoneurial injection of TNF alpha produces neuropathic pain behaviors. Neuro Report 7:2897-2901

48. Wuertz K, Quero L, Sekiguchi M et al (2011) The red wine polyphenol resveratrol shows promising potential for the treatment of nucleus pulposus-mediated pain in vitro and in vivo. Spine 36(21):E1373-E1384 